\title{
SIMULATION OPTIMIZATION USING METAMODELS
}

\author{
Russell R. Barton \\ Department of Supply Chain and Information Systems \\ The Pennsylvania State University \\ 406 Business Building \\ University Park, PA 16803, USA
}

\begin{abstract}
Many iterative optimization methods are designed to be used in conjunction with deterministic objective functions. These optimization methods can be difficult to apply to an objective generated by a discrete-event simulation, due to the stochastic nature of the response(s) and the potentially extensive run times. A metamodel aids simulation optimization by providing a deterministic objective with run times that are generally much shorter than the original discrete-event simulation. Polynomial metamodels generally provide only local approximations, and so a series of metamodels must be fit as the optimization progresses. Other classes of metamodels can provide global fit; fitting can be done either by constructing the global model once at the start of the optimization, or by using the optimization results to identify additional discrete-event runs to refine the global model. This tutorial surveys both local and global metamodel-based optimization methods.
\end{abstract}

\section{INTRODUCTION}

Simulation models provide insight on the behavior of real systems and products. Often the building, verification and validation of a simulation model are followed by ad-hoc exercise of the model to explore "what-if" scenarios. When management objectives can be clearly specified, the simulationist would like to exercise the simulation model with different input parameter settings to find settings that meet the objectives. Simulation optimization is a formal tool for achieving such goals. If we let $Y_{0}$ be the output objective value of the simulation, an element of a possible larger output vector $\boldsymbol{Y}$, and $\boldsymbol{\theta}$ the vector of input parameter settings, the optimization problem formulation can be described in general form as:

$$
\begin{gathered}
\min _{\theta} f(\boldsymbol{\theta}) \equiv E\left(Y_{0}(\boldsymbol{\theta})\right) \\
\text { s.t. } \\
\boldsymbol{a}(\boldsymbol{\theta}) \leq \boldsymbol{b} \\
\boldsymbol{c}(\boldsymbol{Y}(\boldsymbol{\theta})) \leq \boldsymbol{d} .
\end{gathered}
$$

The problem has a stochastic objective if $Y_{0}$ is random. The constraints imposed by the coordinate functions of $\boldsymbol{a}$ and the constraint vector $\boldsymbol{b}$ may allow $\boldsymbol{\theta}$ to take on continuous values or may force one or more components of $\boldsymbol{\theta}$ to be discrete (e.g., integer) valued. If $\boldsymbol{\theta}$ includes time $(t)$ as an element, the optimization has a dynamic response; otherwise it is static. Constraints arising from $\boldsymbol{c}$ are only implicitly known since there is no explicit form for the elements of $\boldsymbol{Y}$ as a function of $\boldsymbol{\theta}$. The simulation optimization problem is unconstrained without the multivariate functions $\boldsymbol{a}$ and $\boldsymbol{c}$.

In spite of this variety, simulation optimization problems have some characteristics in common. The objective is generally not available explicitly, but instead must be estimated by making $R$ replications of the simulation model with parameter vector $\theta_{0}$ say, and estimating the objective by the observed simulation output averaged over the $R$ replications:

$$
f\left(\boldsymbol{\theta}_{0}\right) \cong R^{-1} \sum_{r=1}^{R} Y_{0 r}\left(\boldsymbol{\theta}_{0}\right)
$$




\section{Barton}

Further, the estimation of $f(\boldsymbol{\theta})$ by simulation is often expensive, especially when $R$ must be large. These characteristics have led researchers to develop specialized methods for simulation optimization. These methods were reviewed in April et al. (2003) and Fu et al. (2005).

The authors identify a number of strategies employed for simulation optimization:

- ranking and selection

- metamodel-based methods (he calls RSM)

- gradient-based procedures

- random search

- sample path optimization

- metaheuristics, and a set of

- model-based methods that put a probability distribution on potential solutions.

This tutorial focuses on the second class of optimization strategies; metamodel-based methods for simulation optimization. Methods in this class are relatively easy to implement, and they provide a dual benefit of optimization and insight (Barton and Meckesheimer 2006). The implicitly represented stochastic response of the simulation is replaced by an explicit deterministic response function, as are any implicitly represented constraints. Techniques developed for deterministic optimization can be applied to these metamodel objectives. The next section will describe overall strategies for metamodelbased simulation optimization. The following section will briefly highlight metamodel types and associated properties. The next two sections highlight advances in local and global metamodel-based optimization since Barton and Meckesheimer (2006). The final section summarizes recent events and discusses remaining issues.

\section{METAMODEL-BASED OPTIMIZATION STRATEGIES}

A metamodel-based optimization strategy has the following elements (Barton and Meckesheimer 2006):

- identify a metamodel form

- design an experiment to fit the metamodel

- conduct the simulation experiment

- fit the metamodel and validate the quality of its fit

- optimize the metamodel (or using it to provide a search direction), and

- check the performance of the simulation at the metamodel-predicted optimum (or along the metamodel-determined search direction).

In some cases this process is repeated, with the new experiment design focused on the neighborhood of the predicted optimum. Two general strategies have been used for metamodel-based simulation optimization: global metamodel fit, followed by optimization, and iterated local metamodels. In global metamodel fitting strategies, the entire region of interest (in terms of $\boldsymbol{\theta}$ ) is explored, and the experimental results are used to fit a global approximation. The global approximation is then explored iteratively in the process of optimization. For local fitting strategies, the fitting and optimization steps alternate: as the optimization search moves, new local regions of $\boldsymbol{\theta}$ space are explored, and new metamodel approximations are fitted. Figure 1 shows the differences and similarities between these two classes of fitting strategies.

The local metamodeling strategy is commonly used with low-order polynomial metamodels. The linear or quadratic metamodel is used to determine an optimization search direction. This is followed by a line search, typically evaluating the simulation model directly (averaged over several replications perhaps). Because the metamodels are local, Taylor's Theorem implies that linear and quadratic polynomial models can provide adequate fit. This is the scenario for response surface methodology. Determining the meaning of local is critical to the adequacy of these metamodels and to the success of the method. If the local region is too small, differences in the mean simulation output for different values of $\boldsymbol{\theta}$ will be small relative to the amount of variation in the simulation model output. As a result, the metamodel coefficients will not be sufficiently precise to distinguish them from zero. If the local region is chosen to be too large, linear or quadratic approximations will be inadequate. Local methods thus require checks on metamodel goodness of fit and statistical significance of the metamodel coefficients.

Global metamodel-based optimization is rarely based on polynomial response surface metamodels. Instead, spline, neural network, spatial correlation (kriging) or radial basis function approximations are used. The next section gives a brief overview of these metamodel types and the experiment designs used to fit them. Since global metamodels can have multiple local optima, a global optimization strategy (genetic algorithm, simulated annealing or multistart local optimization) is necessary. 


\section{Barton}

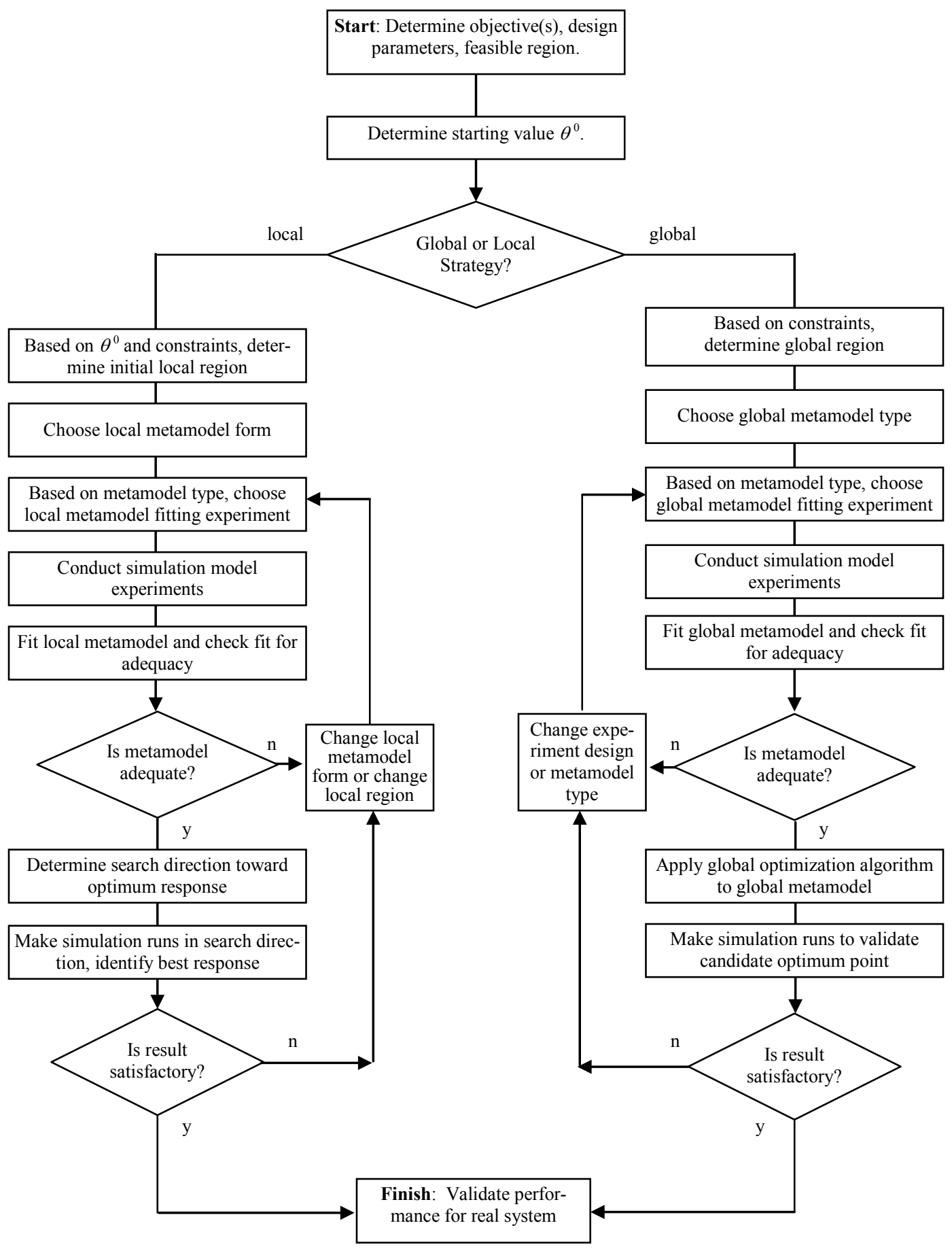

Figure 1: Global vs. local metamodel-based optimization strategy, similarities and differences. 
METAMODEL TYPES

Approximations to simulation input-output response functions have the advantages of i) explicit form, ii) deterministic response and iii) computational efficiency. Since the approximation is a model of a simulation model, Kleijnen called them metamodels (Kleijnen 1975, 2008). They are called surrogate functions in the deterministic simulation community (Yesilyurt and Patera 1995). Running multiple replications of the simulation to produce $f(\theta)$ is expensive; running the metamodel once produces the deterministic value $g(\theta)$ which approximates $f(\theta)$ with low computational expense. The major issues in metamodeling are the choice of a functional form for $g$, the design of experiments, that is, the selection of a set of $\theta$ values at which to observe $Y(\theta)$ by running the simulation model, the assignment of random number streams, the length of runs, etc., the fitting of the metamodel $g$ to the simulation response using the experimental data, and the assessment of the adequacy of the fitted metamodel (confidence intervals, hypothesis tests, lack of fit and other model diagnostics). Many of these issues are discussed in Barton (1998).

Common metamodel functions are shown in Table 1, along with comments on experiment designs and global and local properties. Polynomial models are not appropriate for global approximation in most cases, as explained in Barton (1992) and Barton and Meckesheimer (2006). Kleijnen reviews experiment design strategies for low-order response surface polynomial metamodels (Kleijnen 2007). Yang et al. (2007) use a nonlinear regression metamodel for predicting cycle time as a function of throughput and product mix. Many authors have used the deterministic version of the spatial correlation (kriging) metamodel, but recent papers examine the stochastic version (Huang et al. 2006, Ankenman et al. 2008). The stochastic kriging model represents $Y_{0 r}$ in equation (2) as:

$$
Y_{0 r}(\boldsymbol{x})=g(\boldsymbol{x})^{\prime} \beta+\mathrm{M}(\boldsymbol{x})+\varepsilon_{r}(\boldsymbol{x})
$$

for replication $r$ at design point $\boldsymbol{\theta}=\boldsymbol{x}$, with $\mathrm{M}$ representing the kriging mean component. The model allows modeling the effect of common random numbers, so that $\operatorname{Corr}\left(\varepsilon_{r}(\boldsymbol{x}), \varepsilon_{r}\left(\boldsymbol{x}_{r}{ }^{\prime}\right)\right)>0$. To implement a sequential design strategy for fitting the stochastic kriging model (3), the authors assume statistical independence across runs (no CRN) and introduce a second (but deterministic) kriging metamodel for $V(\boldsymbol{x})=\operatorname{Var}(\varepsilon(\boldsymbol{x}))$. The two-phase strategy allows allocation of replications and new design points in the second phase to minimize an estimated IMSE. The resulting stochastic kriging model need not interpolate average response at the design points.

Van Beers and Kleijnen (2008) introduce a sequential experiment design strategy that can be applied in fitting any global metamodel. The examples fit (deterministic) kriging models to stochastic responses. The strategy is also two-phase, using a pilot maximin or Latin hypercube design. The fit is based on average of $m_{d}$ replicates at $d^{\text {th }}$ design point. These replicate $Y$ values (and those at all other design points) are bootstrap resampled and the kriging model is refitted. With $B$ bootstrap replications, the variance of predicted values at each of $n_{c}$ pre-specified locations is checked. A new design point is added at the location with the highest bootstrap variance.

A piecewise linear metamodel that is a kind of spline uses the simplex interpolation defined in (Weiser and Zarantonello 1988 ) to provide a metamodel response for simulations that can only accept integer values for the elements of $\boldsymbol{\theta}$ (Wang and Schmeiser 2008).

Table 1: Metamodel types, properties and experiment designs for fitting them.

\begin{tabular}{|c|c|c|c|c|}
\hline Metamodel Type & $\begin{array}{c}\text { Local } \\
\text { Approximation }\end{array}$ & Global Approximation & $\begin{array}{c}\text { Experiment } \\
\text { Designs }\end{array}$ & $\begin{array}{c}\text { Stochastic } \\
\text { or Deterministic Response }\end{array}$ \\
\hline $\begin{array}{r}\text { linear and quadratic } \\
\text { polynomial }\end{array}$ & yes & 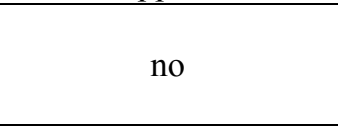 & $\begin{array}{l}\text { fractional factorial } \\
\text { central composite } \\
\text { small composite }\end{array}$ & stochastic \\
\hline $\begin{array}{r}\text { higher order } \\
\text { polynomial }\end{array}$ & no & not recommended & $\begin{array}{c}\text { factorial or } \\
\text { fractional factorial }\end{array}$ & stochastic \\
\hline nonlinear regression & no & phenomenon-specific & factorial & stochastic \\
\hline radial basis function & no & yes & \multirow{3}{*}{$\begin{array}{c}\text { space filling designs: } \\
\text { maximin, orthogonal } \\
\text { array, Latin hypercube } \\
\text { uniform design } \\
\text { application-driven }\end{array}$} & deterministic \\
\hline $\begin{array}{r}\text { spatial } \\
\text { correlation (kriging) }\end{array}$ & no & yes & & $\begin{array}{l}\text { deterministic } \\
\text { or stochastic }\end{array}$ \\
\hline $\begin{array}{r}\text { neural } \\
\text { networks }\end{array}$ & no & yes & & $\begin{array}{l}\text { stochastic or } \\
\text { deterministic }\end{array}$ \\
\hline splines & no & yes & factorial & stochastic or deterministic \\
\hline
\end{tabular}




\section{Barton}

The metamodel types in Table 1 separate into two categories: low-order polynomials for a local metamodel-based optimization strategy (also called response surface methodology), and all other models are for global metamodel-based simulation optimization strategies. Advances in each of these areas are described in the next two sections.

\section{RESPONSE SURFACE METHODOLOGY}

Response surface methodology (RSM) has its origins in the work of Box and Wilson (1951). They developed the approach while working with a company to determine optimal operating conditions for chemical processes. One of the earliest applications in simulation was by Biles (1974). A current comprehensive reference for RSM is Myers et al. (2009). Formal RSM algorithms for metamodel-based simulation optimization are described in Neddermeijer et al. (2000) and Nicolai et al. (2004). They follow the general structure in the left half of Figure 1. A formal RSM procedure following this structure is also described in Barton and Meckesheimer (2006). Despite its long history, there have been continuing developments in RSM in recent years. These are summarized in the paragraphs below, and later in Table 2.

Kleijnen et al. (2004) proposed an alternative to the steepest descent search direction, and step length. In a minimization setting, the method searches for the parameter $\boldsymbol{\theta}^{\prime}$ that gives the lowest upper confidence interval on predicted mean performance. The search direction is then from the current point toward $\boldsymbol{\theta}^{\prime}$.

Oon and Lee (2006) investigated the advantage of ordinal optimization in RSM. In place of the simulation response $Y$, the rank of each $Y$ was used as the dependent variable for the regression model fit. In some cases their ordinal RSM performed comparably to RSM based on actual values, but their conclusion was that it was inferior to ordinary RSM.

Chang et al. (2007) incorporated the trust region concept from unconstrained optimization in RSM. Sequential quadratic approximations are fitted using orthogonal experiment designs. An optimization step is determined based on the quadratic model, with magnitude limit based on the trust region size. The simulation model is evaluated at this new point, followed by a check comparing the actual reduction against the reduction predicted by the quadratic approximation. If the reduction is a small fraction of the amount predicted, the size of the trust region is decreased. If the actual and predicted decrease are nearly the same, the trust region size is increased. Otherwise the trust region size remains unchanged. If the improvement is not statistically significant, additional simulation replications are allocated.

RSM is generally applied in unconstrained optimization settings. Kleijnen and co-authors have formally extended the method to incorporate constraints. Biles et al. (2007) presented some examples. The approach is based on constructing hypothesis tests for the plausibility of the Karush-Kuhn-Tucker conditions of nonlinear programming at the candidate optimal solution. The details were presented in Bettonvil et al. (2009), and the method was summarized in Kleijnen (2008c).

Many of these issues in response surface methodology were presented in del Castillo (2007), in some cases with additional (and better) alternatives.

\section{GLOBAL METAMODEL-BASED SIMULATION OPTIMIZATION}

The flexibility of neural networks, radial basis functions, splines and spatial correlation (kriging) models present an opportunity to fit and optimize a single metamodel, eliminating the need to repeatedly design experiments, make runs and fit a sequence of local metamodels. This removes the need for sequential decisions on the type of metamodel to be fit and the kind of experiment design to be used for fitting, and allows more complete automation of the optimization process. Global metamodel-based simulation does not require a simultaneous design strategy, however. More complex global metamodel-based optimization methods update the global fit by selecting additional simulation runs as the optimization progresses (Alexandrov et al. 1998, Jones et al. 1998, Booker et al. 1999). This section reviews recent developments in global metamodel-based simulation optimization, extending the discussion in Barton and Meckesheimer (2006).

Compared with the nearly sixty-year history of RSM, global simulation metamodel optimization has been an active area for less than twenty years. Barton (1992) summarized many global approximation models and their potential application in simulation metamodeling. The earliest focus was on modeling, not optimization, and initial optimization work was for deterministic simulations such as finite element and circuit simulation models (Bernardo et al. 1992; later reviews include Simpson et al. 2001, Simpson et al. 2004 and Samarasinghe 2006). This setting has come to be known as DACE, or the design and analysis of computer experiments (Santner et al. 2003).

Wang (2005) proposed a formal neural network metamodel-based simulation optimization procedure. Neural networks can be used to fit either deterministic or stochastic responses, which adds flexibility to the method. The (unconstrained) optimization is performed using a genetic algorithm (GA). The GA approach can require many function evaluations, but these are performed using the metamodel, minimizing the computational cost.

While applications of global metamodeling methods such as neural networks and radial basis functions have received some attention, the most active area of recent research in metamodel-based simulation optimization has centered around spa- 


\section{Barton}

tial correlation models. The spatial correlation papers of Sacks et al. (1989), Currin et al. (1991), Handcock and Stein (1993), Morris et al. (1993) and Cressie (1993) among others laid out a framework for fitting and analyzing spatial correlation models. These studies expanded on the spatial correlation model of Krige (1951), formalized by Matheron (Matheron 1962, 1963), and so are frequently called kriging metamodels.

Recent advances in kriging metamodel-based optimization extend the frameworks of Alexandrov, Jones et al. and Booker. Formal methods for kriging metamodel-based optimization with constraints were proposed by Sasena et al. (2002), Biles et al. (2007) and Kleijnen et al. (2009). Sasena et al. focused on deterministic simulation response functions. Biles et al. and Kleijnen et al. used the interpolating (deterministic) version of the kriging metamodel fitted to the outputs of a stochastic simulation. These metamodels are used as approximations to objective and constraint functions, and a nonlinear programming optimization method is applied to the approximations. If the optimization yields a new candidate $\theta^{*}$, then the original simulation code is exercised at $\boldsymbol{\theta}^{*}$. The algorithm includes validity and termination criteria. Allen et al. (2003) noted that bias error in metamodels often dominates the error introduced by a stochastic objective, and Kleijnen and co-authors used the same argument to justify the deterministic (interpolating) kriging form.

Huang et al. (2006) introduced a stochastic kriging metamodel-based optimization method. The kriging model follows the form in Equation (3). The sequential experiment design strategy used a sophisticated approach, based on the Jones et al. expected improvement function. The expected improvement function is augmented to capture uncertainty from the stochastic response. The method performed well on five test cases. One drawback cited by the authors was the significant computational time needed to fit the kriging models. The method and test cases were unconstrained optimizations, but the authors suggested that the method could be extended to constrained optimization using the approach described in Sasena et al.

\section{SUMMARY}

Table 2 summarizes the recent developments in metamodel-based simulation optimization described in the last two sections. It only includes complete methods, not improvements related to individual components of an overall metamodel-based simulation optimization strategy. More details on metamodel-based simulation optimization can be found in Barton and Meckesheimer (2006), Kleijnen (2008a), and Kleijnen (2008b).

This survey has excluded likelihood ratio, mathematical programming and score function sensitivity methods, which might be viewed as kinds of metamodel-based optimization. Rubinstein and Shapiro (1993), L'Ecuyer and Glynn (1994), Kleijnen and Rubinstein (1996), Rubinstein and Melamed (1998) and Chan and Schruben (2006) all examine simulation optimization strategies based on local derivative estimates. Convergence results for optimization are presented in Rubinstein and Shapiro (1993).

This overview indicates that there has been significant progress in recent years in metamodel-based simulation optimization methods, but much remains to be done. While many extensions and improvements have been developed, few convergence results exist (but see del Castillo 2007). Further, many methods require decision procedures that are difficult to fully automate. Finally, the relative performance of competing metamodel-based simulation optimization strategies remains unclear. Comparisons have been informal, ad-hoc and not comprehensive. The future is bright for continuing research in this area.

Table 2: Summary of recent developments in metamodel-based simulation optimization.

\begin{tabular}{|c|c|c|c|c|c|c|}
\hline & $\begin{array}{c}\text { Metamodel } \\
\text { Type(s) }\end{array}$ & \begin{tabular}{|c|} 
Response \\
modeled as \\
Deterministic \\
or Stochastic
\end{tabular} & $\begin{array}{c}\text { Continuous, In- } \\
\text { teger or Mixed } \\
\text { Decision Va- } \\
\text { riables }\end{array}$ & $\begin{array}{c}\text { Constrained or } \\
\text { Unconstrained } \\
\text { Optimization }\end{array}$ & $\begin{array}{c}\text { Simultaneous } \\
\text { or Sequential } \\
\text { DOE }\end{array}$ & $\begin{array}{c}\text { Local } \\
\text { Models or } \\
\text { Global } \\
\text { Model } \\
\end{array}$ \\
\hline \multicolumn{7}{|l|}{ Paper } \\
\hline Kleijnen 2008 & $\begin{array}{l}\text { Linear \& } \\
\text { Quadratic }\end{array}$ & Stochastic & Continuous & Constrained & Sequential & Local \\
\hline Chang et al. 2007 & $\begin{array}{l}\text { Linear \& } \\
\text { Quadratic }\end{array}$ & Stochastic & Continuous & Unconstrained & Sequential & Local \\
\hline Oon and Lee 2006 & $\begin{array}{l}\text { Linear \& } \\
\text { Quadratic }\end{array}$ & $\begin{array}{c}\text { Stochastic } \\
\text { (Rank) }\end{array}$ & Continuous & Unconstrained & Sequential & Local \\
\hline Biles et al. 2007; Kleijnen et al. 2009 & Kriging & Deterministic & Continuous & Constrained & Simultaneous & Global \\
\hline Huang et al. 2006 & Kriging & Stochastic & Continuous & Unconstrained & Sequential & Global \\
\hline Wang 2005 & $\begin{array}{c}\text { Neural } \\
\text { Network }\end{array}$ & Deterministic & Continuous & Constrained & Simultaneous & Global \\
\hline
\end{tabular}




\section{Barton}

\section{REFERENCES}

Alexandrov, N. M., J. E. Dennis, Jr., R. M. Lewis and V. Torczon. 1998. A trust region framework for managing the use of approximation models in optimization. Structural Optimization 15(1): 16-23.

Allen, T. T., M. A. Bernshteyn and K. Kabiri-Bamoradian. 2003. Constructing meta-models for computer experiments. Journal of Quality Technology 35(3): 264-274.

Ankenman, B., B. L. Nelson and J. Staum. 2008. Stochastic kriging for simulation metamodeling. In Proceedings of the 2008 Winter Simulation Conference, ed. S. J. Mason, R. R. Hill, L. Mönch, O. Rose, T. Jefferson, J. W. Fowler, 362-370. Piscataway, New Jersey: Institute of Electrical and Electronics Engineers.

April, J. F. Glover, J. P. Kelly and M. Laguna. 2003. Simulation-based optimization: practical introduction to simulation optimization. In Proceedings of the 2003 Winter Simulation Conference, ed. S. Chick, P. J. Sánchez, D. Ferrin, and D. J. Morrice, 71-78. Piscataway, New Jersey: Institute of Electrical and Electronics Engineers.

Barton, R. R. 1992. Metamodels for simulation input-output relations. In Proceedings of the 1992 Winter Simulation Conference, ed. J. J. Swain, D. Goldsman, R. C. Crain, and J. R. Wilson, 289-299. Piscataway, New Jersey: Institute of Electrical and Electronics Engineers.

Barton, R. R. 1998. Simulation metamodels. In Proceedings of the 1998 Winter Simulation Conference, ed. D.J. Medeiros, E.F. Watson, J.S. Carson and M.S. Manivannan, 167-174. Piscataway, New Jersey: Institute of Electrical and Electronics Engineers.

Barton, R. R. and M. Meckesheimer. 2006. Metamodel-based simulation optimization. In Simulation. Handbooks in Operations Research and Management Science vol. 13. Amsterdam: Elsevier B.V.

van Beers, W. C. M. and J. P. C. Kleijnen. 2008. Customized sequential designs for random simulation experiments: kriging metamodeling and bootstrapping. European Journal of Operational Research 186(3): 1099-1113.

Bernardo, M. C., R. Buck, L. Liu, W. A. Nazaret, J. Sacks and W. J. Welch. 1992. Integrated circuit design optimization using a sequential strategy. IEEE Transactions on Computer-Aided Design of Integrated Circuits and Systems 11(3): 361372.

Bettonvil, B., E. del Castillo and J. P. C. Kleijnen. 2009. Statistical testing of optimality conditions in multiresponse simulation-based optimization. European Journal of Operational Research 199(2): 448-458.

Biles, W. E. 1974. A gradient regression search procedure for simulation experimentation. In Proceedings of the 1974 Winter Simulation Conference, ed. H. Highland, H. Steinberg and M. F. Morris, 491-497. Piscataway, New Jersey: Institute of Electrical and Electronics Engineers.

Biles, W. E., J. P. C. Kleijnen, W. C. van Beers and I. Nieuwenhuyse. 2007. Kriging metamodeling in constrained simulation optimization: an explorative study. In Proceedings of the 2007 Winter Simulation Conference, ed. S. G. Henderson, B. Biller, M.-H. Hsieh, J. Shortle, J. D. Tew, and R. R. Barton, 355-362. Piscataway, New Jersey: Institute of Electrical and Electronics Engineers.

Booker, A. J., J. E. Dennis, Jr., P. D. Frank, D. B. Serafini, V. Torczon and M. W. Trosset. 1999. A rigorous framework for optimization of expensive functions by surrogates. Structural and Multidisciplinary Optimization 17(1): 1-13.

Box, G. E. P. and K. B. Wilson. 1951. On the experimental attainment of optimum conditions. Journal of the Royal Statistical Society. Series B (Methodological) 13: 1-45.

del Castillo, E. D. 2007. Process optimization. New York: Springer.

Chan, W. K. and L. W. Schruben. 2006. Response gradient estimation using mathematical programming models of discreteevent system sample paths. In Proceedings of the 2006 Winter Simulation Conference, ed. L. F. Perrone, F. P. Wieland, J. Liu, B. G. Lawson, D. M. Nicol, and R. M. Fujimoto, 272-278. Piscataway, New Jersey: Institute of Electrical and Electronics Engineers.

Chang, K., L. J. Hong and H. Wan. 2007. Stochastic trust region gradient-free method (strong): a new response-surface-based algorithm in simulation optimization. In Proceedings of the 2007 Winter Simulation Conference, ed. S. G. Henderson, B. Biller, M.-H. Hsieh, J. Shortle, J. D. Tew, and R. R. Barton, 346-354. Piscataway, New Jersey: Institute of Electrical and Electronics Engineers.

Cressie, N. A. C. 1993. Statistics for spatial data. New York: John Wiley and Sons.

Currin, C., T. Mitchell, M. Morris and D. Ylvisaker. 1991. Bayesian prediction of deterministic functions, with applications to the design and analysis of computer experiments. Journal of the American Statistical Association 86(416): 953-963.

Fu, M., F. Glover and J. April. 2005. Simulation optimization: a review, new developments, and applications. In Proceedings of the 2005 Winter Simulation Conference, ed. M. E. Kuhl, N. M. Steiger, F. B. Armstrong, and J. A. Joines, 83-95. Piscataway, New Jersey: Institute of Electrical and Electronics Engineers.

Handcock, M. S. and M. L. Stein. 1993. A Bayesian analysis of kriging. Technometrics 35(4): 403-410. 


\section{Barton}

Huang, D., T. T. Allen, W. Notz and N. Zeng. 2006. Global optimization of stochastic black-box systems via sequential kriging meta-models. Journal of Global Optimization 34: 441-466.

Jones, D., M. Schonlau and W. Welch. 1998. Efficient global optimization of expensive black-box functions. Journal of Global Optimization 13: 455-492.

Kleijnen, J. P. C. 1975. A comment on Blanning's "metamodel for sensitivity analysis: the regression metamodel in simulation." Interfaces 5(3): 21-23.

Kleijnen, J. P. C. 2007. Regression models and experimental designs: a tutorial for simulation analysts. In Proceedings of the 2007 Winter Simulation Conference, ed. S. G. Henderson, B. Biller, M.-H. Hsieh, J. Shortle, J. D. Tew, and R. R. Barton, 183-194. Piscataway, New Jersey: Institute of Electrical and Electronics Engineers.

Kleijnen, J. P. C. 2008a. Design and analysis of simulation experiments. New York: Springer.

Kleijnen, J. P. C. 2008b. Design of experiments: overview. In Proceedings of the 2008 Winter Simulation Conference, ed. S. J. Mason, R. R. Hill, L. Mönch, O. Rose, T. Jefferson, J. W. Fowler, 479-488. Piscataway, New Jersey: Institute of Electrical and Electronics Engineers.

Kleijnen, J. P. C. 2008c. Response surface methodology for constrained simulation optimization: an overview. Simulation Modelling Practice and Theory 16: 50-64.

Kleijnen, J. P. C., W. C. M. van Beers and I. Nieuwenhuyse. 2009. Constrained optimization in simulation: a novel approach. European Journal of Operational Research (to appear).

Kleijnen, J. P. C., D. den Hertog and E. Angun. 2004. Response surface methodology's steepest ascent and step size revisited. European Journal of Operational Research 159(1): 121-131.

Kleijnen, J. P. C. and R. Y. Rubinstein. 1996. Optimization and sensitivity analysis of computer simulation models by the score function method. European Journal of Operational Research 88: 413-427.

Krige, D.G. 1951. A statistical approach to some basic mine valuation problems on the Witwatersrand. Journal of the Chemical, Metallurgical and Mining Society of South Africa 52(6): 119-139.

L'Ecuyer, P. and P. W. Glynn. 1994. Stochastic optimization by simulation: convergence proofs for the GI/G/1 queue in steady-state. Management Science 40(11): 1562-1578.

Matheron, G. 1962. Traité de Géostatistique Appliquée, Tome 1, Paris: Technip.

Matheron, G. 1963. Traité de Géostatistique Appliquée, Tome 2, Paris: Technip.

Morris, M. D., T. J. Mitchell and D. Ylvisaker. 1993. Bayesian design and analysis of computer experiments: use of derivatives in surface prediction. Technometrics 35(3): 243-255.

Myers, R. H., D. C. Montgomery and C. M. Anderson-Cook. 2009. Response surface methodology. New York: John Wiley and Sons.

Neddermeijer, H. G., G. J. van Oortmarssen, N. Piersma and R. Dekker. 2000. A framework for response surface methodology for simulation optimization. In Proceedings of the 2000 Winter Simulation Conference, ed. J. A. Joines, R. R. Barton, K. Kang, and P. A. Fishwick, 129-136. Piscataway, New Jersey: Institute of Electrical and Electronics Engineers.

Nicolai, R. P., R. Dekker, N. Piersma and G. J. van Oortmarssen. 2004. Automated response surface methodology for stochastic optimization models with unknown variance. In Proceedings of the 2004 Winter Simulation Conference, ed. R. G. Ingalls, M. D. Rossetti, J. S. Smith, and B. A. Peters, 491-499. Piscataway, New Jersey: Institute of Electrical and Electronics Engineers.

Oon, S. J. and L. H. Lee. 2006. The impact of ordinal on response surface methodology. In Proceedings of the 2006 Winter Simulation Conference, ed. L. F. Perrone, F. P. Wieland, J. Liu, B. G. Lawson, D. M. Nicol, and R. M. Fujimoto, 406413. Piscataway, New Jersey: Institute of Electrical and Electronics Engineers.

Rubinstein, R. Y. and B. Melamed. 1998. Modern simulation and modeling. 1st ed. New York: Wiley-Interscience.

Rubinstein, R. Y. and A. Shapiro. 1993. Discrete event systems. New York: John Wiley and Sons.

Sacks, J., W. J. Welch, T. J. Mitchell and H. P. Wynn. 1989. Design and analysis of computer experiments. Statistical Science 4(4): 409-423.

Samarasinghe, S. 2006. Neural networks for applied sciences and engineering. New York: CRC Press.

Santner, T. J., B. J. Williams and W. I. Notz. 2003. The design and analysis of computer experiments, new York: Springer.

Sasena, M. J., P. Papalambros and P. Goovaerts. 2002. Exploration of metamodeling sampling criteria for constrained global optimization. Engineering Optimization 34: 263-278.

Simpson, T. W., A. J. Booker, D. Ghosh, A. A. Giunta, P. N. Koch and R.-J. Yang. 2004. Approximation methods in multidisciplinary analysis and optimization: a panel discussion. Structural and Multidisciplinary Optimization 27(5): 302-313.

Simpson, T. W., J. J. Korte, T. Mauery and F. Mistree. 2001. Kriging models for global approximation in simulation-based multidisciplinary design optimization. AIAA Journal 39: 2233-2241. 


\section{Barton}

Wang, H. and B. W. Schmeiser. 2008. Discrete stochastic optimization using linear interpolation. In Proceedings of the 2008 Winter Simulation Conference, ed. S. J. Mason, R. R. Hill, L. Mönch, O. Rose, T. Jefferson, J. W. Fowler, 502-508. Piscataway, New Jersey: Institute of Electrical and Electronics Engineers.

Wang, L. 2005. A hybrid genetic algorithm-neural network strategy for simulation optimization. Applied Mathematics and Computation 170(2): 1329-1343.

Weiser, A. S. E. Zarantonello. 1988. A note on piecewise linear and multilinear table interpolation in many dimensions. $M a-$ thematics of Computation 50(181): 189-196.

Yang, F., J. Liu, M. Tongarlak, B. E. Ankenman and B. L. Nelson. 2007. Metamodeling for cycle time-throughput-product mix surfaces using progressive model fitting. In Proceedings of the 2007 Winter Simulation Conference, ed. S. G. Henderson, B. Biller, M.-H. Hsieh, J. Shortle, J. D. Tew, and R. R. Barton, 322-330. Piscataway, New Jersey: Institute of Electrical and Electronics Engineers.

Yesilyurt, S. and A. T. Patera. 1995. Surrogates for numerical simulations; optimization of eddy-promoter heat exchangers. Computer Methods in Applied Mechanics and Engineering 121: 231-257.

\section{AUTHOR BIOGRAPHY}

RUSSELL R. BARTON is a professor in the Department of Supply Chain and Information Systems at the Pennsylvania State University. He is co-director of the Penn State Master of Manufacturing Management degree program and associate director of the Center for the Management of Technological and Organizational Change. He received a B.S. degree in Electrical Engineering from Princeton University and M.S. and Ph.D. degrees in Operations Research from Cornell University. Before entering academia, he spent twelve years in industry. He is a past president of the INFORMS Simulation Society and serves on the advisory board for the INFORMS Quality Statistics and Reliability section. He is a senior member of IIE and IEEE. His research interests include applications of statistical and simulation methods to system design and to product design, manufacturing and delivery. His email address is <rbarton@psu.edu>. 